\title{
Kompetenzzentrum erschließt 3D-Druck für die Feuerfestindustrie
}

\begin{abstract}
Die additive Fertigung hat in den letzten Jahrzehnten in vielen Branchen stark an Bedeutung gewonnen. Im Kompetenzzentrum für den 3D-Druck von Keramik in Höhr-Grenzhausen sollen erstmals feuerfeste Formteile hergestellt werden. Für die Aufbereitung der Massen fiel die Wahl auf einen Mischer von Eirich.
\end{abstract}

Kein anderes Formgebungsverfahren für Bauteile aus Kunststoff, Keramik oder Metall bietet so viel Potenzial für Kosteneinsparungen wie der 3D-Druck - schnellere Produktion, geringere Werkzeugkosten, weniger Arbeitsschritte und weniger Ausschuss. Auch bei der Prototypenherstellung und Kleinserienfertigung ergeben sich deutliche Einsparungen, neben einer höheren Materialeffizienz und einer Gewichtsersparnis. In der Keramik hat sich der 3D-Druck lange Zeit auf kleine Bauteile fokussiert, die konventionell nur aufwändig herzustellen waren, und so hat sich das Verfahren in der technischen Keramik und Dentalkeramik inzwischen erfolgreich durchgesetzt. Doch während in der Bauindustrie heute als Vorzeigeobjekte ganze Häuser in additiver Fertigung erstellt werden, hat die Keramische Industrie bei größeren Bauteilen und Objekten noch Nachholbedarf.

\section{Fertigungskompetenz aus Höhr-Grenzhausen}

Die Landesregierung Rheinland-Pfalz hat das Potenzial erkannt. In der Folge wurde im Sommer 2019 der Aufbau eines Kompetenzzentrums für den 3D-Druck von Keramik in Höhr-Grenzhausen mit rund 5,3 Millionen Euro gefördert. Das Zentrum wird vom Forschungsinstitut für Anorganische Werkstoffe -Glas/Keramik (FGK), der Hochschule Koblenz und der Universität Koblenz-Landau eingerichtet. Das Projekt dient zunächst zur Entwicklung einer Infrastruktur und dem Aufbau eines Knowhows zur additiven Fertigung von anorganisch-nichtmetallischen Werkstoffen. Diese erstrecken sich über die gesamte Bandbreite keramischer Werkstoffe - von der Silikatkeramik über die Feuerfestkeramik, die technische Keramik und Transparentkeramik bis hin zum Multimaterialdruck.

\section{Skalierbarer Mischer für feuerfeste Massen}

Für die Aufbereitung der Feuerfestmassen fiel die Entscheidung auf einen 75-1-Mischer von Eirich, der sich besonders dadurch auszeichnet, dass ein sich drehender Mischbehälter das Mischgut zum Mischwerkzeug transportiert. Der Transport des Mischguts ist so vom eigentlichen Mischvorgang entkoppelt und schnelllaufende, am Boden oder nahe der Behälterwand angeordnete und damit verschleißintensive Werkzeuge entfallen. Dies führt dazu, dass die Mischwerkzeuge schneller laufen können, ohne übermäßig Reibung und Verschleiß am Mischbehälter zu bewirken.

Ein weiteres Merkmal ist die Scale-upFähigkeit. Wenn Mischungen auf kleinen Mischern entwickelt werden, ist die Übertragung auf größere Mischer oft problematisch. Bei Schubmischern (wie z. B. Ringtrog- und Planetenmischern) und bei Wurfmischern (z. B. Horizontalmischern) werden zusätzliche Werkzeugsätze benötigt, wenn der Mischer größer wird, womit sich die Leistungsdichte im Mischbehälter ändert. Beim Horizontalmischer sind zudem zur Unterstützung des Mischprozesses und zur Desagglomeration von Feinststoffen schnell laufende Messerköpfe erforderlich, deren Wirkung wiederum nicht scale-up-fähig ist. In den Mischern von Eirich zwischen 11 und 30001 Fassungsvermögen wird nur ein einziges Mischwerkzeug benötigt, der Wirbler, über den sich der Leistungseintrag in die Mischung durch die Werkzeuggeschwindigkeit sowie die Drehrichtung sehr genau kontrollieren lässt. Der neue Mischer

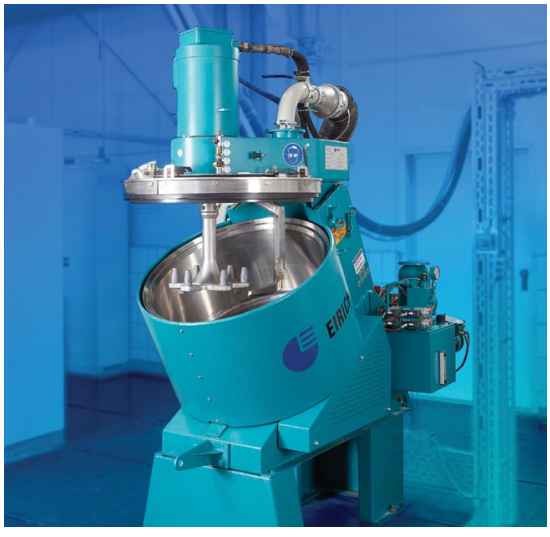

Der neue 75-I-Mischer für die Aufbereitung feuerfester Massen wird im Frühjahr 2021 geliefert. (๔ Eirich)

wird im Frühjahr 2021 an die Hochschule geliefert. Als Besonderheit ist hier nicht nur der Wirblerantrieb mitFU-Geschwindigkeitsregelung ausgeführt, sondern auch der Mischbehälter. Damit entstehen zusätzliche Freiheitsgrade in der Prozessführung.

Für die Hochschule Koblenz bedeutet das neue Kompetenzzentrum eine weitere Stärkung des WesterwaldCampus in HöhrGrenzhausen als innovativer Forschungsstandort. Dort ist auch das ECREF - European Centre for Refractories zuhause, welches das internationale Feuerfestkolloquium (ICR) in Aachen ausrichtet. Mit dem 3D-Druck-Kompetenzzentrum nutzt die Feuerfestindustrie in Höhr-Grenzhausen eine Technologie, die wirtschaftlich und damit zukunftsweisend ist.

Kontakt

Maschinenfabrik Gustav Eirich, Hardheim, www.eirich.de;

Hochschule Koblenz, WesterwaldCampus, www.hs-koblenz.de/wwc 